\section{Endosonographic Diagnosis of Duodenal Diverticulum Mimicking a Pancreatic Tumor}

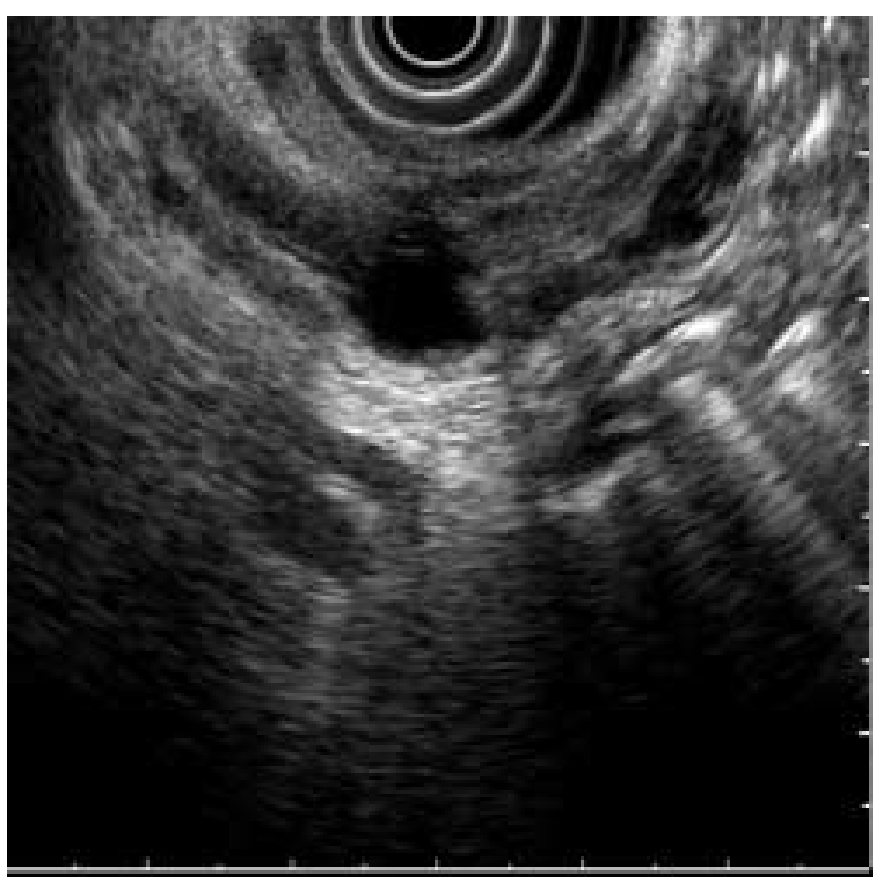

Figure 1 A 79-yearold woman presented for evaluation of a pancreatic head mass lesion that had been evident on abdominal ultrasound, which had been performed because of nonspecific upper abdominal pain. Endoscopic ultrasound (EUS) initially visualized an inhomogeneous, partially echo-free lesion in the periampullary area next to the duodenal wall.

Figure 2 On water instillation into the duodenum, the lesion $\left({ }^{*}\right)$ gradually filled with water, displaying wall structures similar to those of the gastrointestinal wall, with the slightly dilated common bile duct (CBD) running towards the water-filled "lesion". The endosonographic diagnosis of a periampullary diverticulum was thus very likely to be accurate. The pancreatic head was otherwise normal. PV, portal vein.

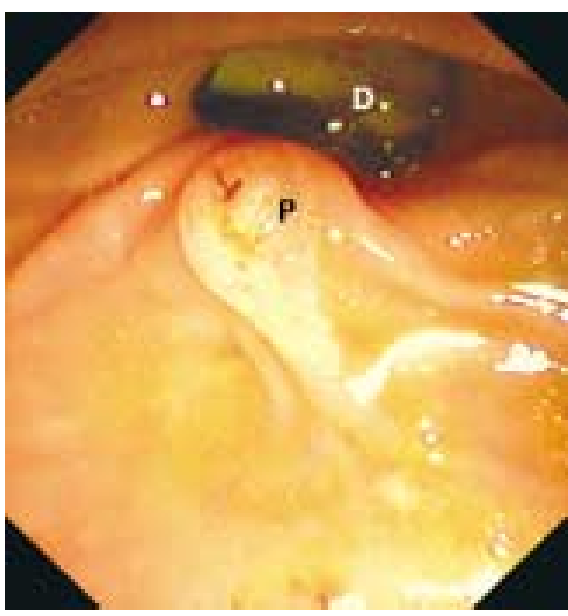

Figure 3 This was confirmed by side-viewing endoscopy ( $D$, diverticulum; $P$, papilla). The view of the papilla is usually less well seen with the oblique optics of echo endoscopy, and so this aspect was initially not evident when EUS was performed. Awareness of this possibility is therefore crucial at EUS, in order to avoid making a false diagnosis of pancreatic mass lesion.

\section{P. Born, T. Rösch}

Department of Internal Medicine II, Technical University of Munich, Klinikum rechts der Isar, Munich, Germany

\section{Corresponding Author}

\section{P. Born, M.D.}

Department of Internal Medicine II Klinikum rechts der Isar Technical University of Munich Ismaningerstr. 22

81675 München

Germany

Fax: $\quad+49-89-4140-4905$

E-mail: Peter.Born@lrz.tum.de 